\title{
Towards an intelligent system for FMS design
}

\author{
Babic B.R. \\ University of Belgrade, Faculty of Mechanical Engineering \\ 27. Marta 80, 11000 Belgrade, Yugoslavia; phone: 381113229 040; \\ fax: 381113221 198; e-mail:mlbabic@rcub.rcub.bg.ac.yu
}

\begin{abstract}
The paper presents the concept of an intelligent system performing creative FMS design. Development of the intelligent system for FMS design is based on the application of two theories: design theory and theory of reasoning by analogy. Described general design process is based on design axioms. In accordance with axiomatic design concept four levels of intelligent system for FMS design are defined: functional requirements (FRs) definition level, design parameters (DPs) creation level, the level for analysis of design solution, and the level for checking of the final solution. For each level a particular knowledge processing module is defined. Computer software dealing with each one of four levels is also discussed.
\end{abstract}

\section{Keywords}

Flexible Manufacturing Systems, Design Axioms, Knowledge Processing.

\section{INTRODUCTION}

The design of manufacturing systems, particularly of flexible manufacturing systems (FMS) is a complex problem that includes the planning of capacities, balancing of operations, transport analysis, storage planning and the like. Adequate solution of some manufacturing problem requires a comprehensive study of all these factors (in their interaction). Present approaches in the field of computer-aided engineering design, applicable for the FMS design, are mainly based on data processing technology. Since data represent specific instances of events, it is not so easy to deal with various situations occurring during the FMS design. To improve productivity of FMS design process it is essential to set up a new concept based on the knowledge processing.

The paper gives basic principles used for development of the intelligent system for FMS design (FLEXY). The development of an intelligent system for FMS design is based on the integration of two theories: design theory and theory of reasoning by analogy. For the formalization of FMS design process axiomatic design theory established by Prof. Nam P. Suh 
(MIT) was adopted. Details of this theory are given in (Suh, 1990) and (Kim, 1991) and only a short overview is given in this paper.

Designers most commonly apply experiences gathered through previous projects to a new project through analogy. The introduction of reasoning by analogy into the FMS design system should bring creative component to the system. Determinism based formalization of reasoning by analogy, given in (Russell, 1989), is applied for the building of FLEXY system. Computer software corresponding to the proposed model of intelligent system for FMS design was developed in PROLOG and tested on several examples.

\section{DESIGN THEORY}

By the axiomatic design theory the design is formally defined as the creation of synthesized solutions in the form of products, processes or systems that satisfy perceived needs through the mapping between the functional requirements (FRs) in the functional domain and the design parameters (DPs) of physical domain, through proper selection of DPs that satisfy FRs (Suh, 1990). This is illustrated in Figure 1. The design objective is always stated in the functional domain, whereas the physical solution is always generated in physical domain. The mapping process is non unique; therefore, there can be an infinite number of plausible design solutions and mapping techniques. The design axioms provide the principles that the mapping technique must satisfy to produce a good design, and offer a basis for comparing and selecting designs.

Design is defined as mapping process from the functional space to the physical space.

The following two axioms are used:

Axiom 1: The Independence Axiom

Maintain the independence of functional requirements

Axiom 2: The Information Axiom

Minimize the information content

Axiom 1 distinguishes good and bad design, or acceptable and unacceptable solution. Axiom 2 is the criterion for selection of the optimum design solution from those that satisfy

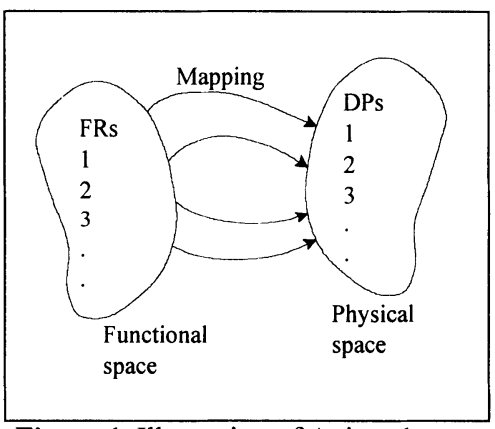

Figure 1 Illustration of Axiom 1. Axiom 1 (Suh, 1990).

There are some propositions that follow from axioms. These propositions are called corollaries and they represent specific design rules. Here, the corollaries relevant for FMS design are only given:
Corollary 2 Minimization of FRs
Corollary 3
Integration of Physical Parts
Corollary 6 Largest Tolerance
Corollary 7 Uncoupled Design with Less Information

\section{AXIOMATIC DESIGN OF FMS}

The general design theory given above is concretized for the FMS design. This is illustrated in Figure 2. The design process begins with the establishment of FRs in the functional domain to satisfy a given set of needs. The needs that FMS design should satisfy are defined by 
the set of workpieces that should be produced, batch sizes, due dates, etc. Needs specification with corresponding process plans is the basis for the formation of FRs. FRs are expressed in the following form:

FRs (Machining_type; Machining_Accuracy; Max_Surface_Roughness;

Max_Workpiece_Dimension; Machining_Operation)

There are also FRs dealing with due dates. These FRs have the following form:

FRs (Work_piece; Due_date)

The beginning set of FRs is reduced in accordance with corollary 2 . The next step of axiomatic FMS design is mapping between functional domain and physical domain.

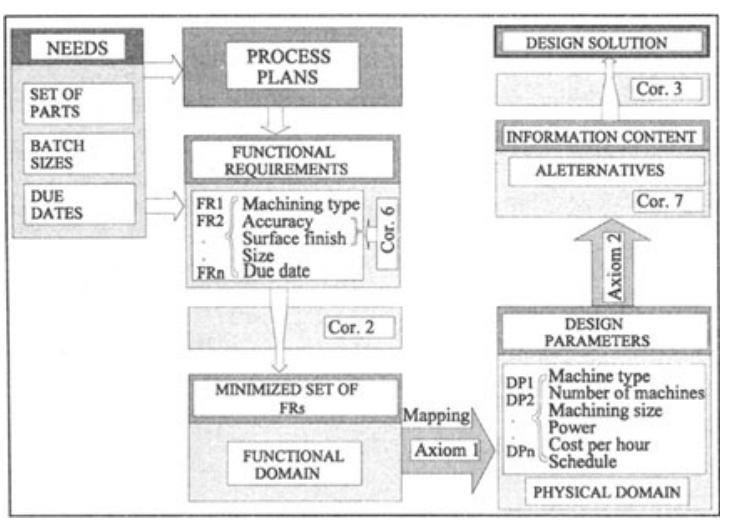

Figure 2 Axiomatic design of FMS.

As mentioned in previous section, the mapping process is not unique, therefore, more than one design may ensue for generation of the DPs that satisfy FRs. The information axiom and corollary 7 provide criterion for selection of the optimum design solution. Information content is calculated on the basis of the following equation (Suh,1990):

$$
I=\frac{\text { system range }}{\text { common range }}(1)
$$

The system range is the capability of the current manufacturing system, whereas the design range is the tolerance specified by designer. The common range is the overlap area between the system range and the design range. Information content of FMS is sum of the following partial information contents: information content dealing with geometrical accuracy, information content dealing with surface quality, information content dealing with production capacity, information content dealing with production costs.

Final design solution is reached through the application of corollary 3. Corollary 3 states that integration of physical parts should be done, if possible. In the case of FMS design machining stations performing similar operations are integrated (Babic,1993).

\section{ARCHITECTURE OF FLEXY SYSTEM}

Theoretical considerations given in previous sections are the basis for the definition of FLEXY architecture. In accordance with the axiomatic design concept, the architecture of intelligent system for FMS design must involve the following four levels: functional requirements (FRs) definition level, design parameters (DPs) creation level, the level for analysis of design solution, and the level for checking of the final solution

At the first level, a set of FRs are defined in the functional domain in order to satisfy perceived needs. Then, a set of DPs are defined in order to satisfy the FRs defined at level 1. After this proposed solutions are analyzed for acceptability. Finally, the fidelity of the final solution to the originally perceived needs is checked. 


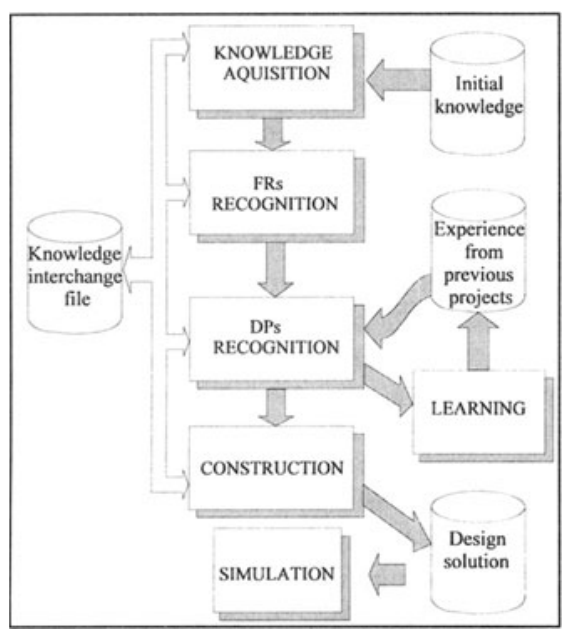

Figure 7 Architecture of FLEXY system.

:- Module FMS_DM

Reconsult ('Base.Ari'),

Reconsult ('Analog.Ari'),

Load base,

$F R$ recog,

The instruction Reconsult ('Base.Ari') opens a file containing initial knowledge defined. The next instruction Reconsult ('Analog.Ari') opens a space of knowledge from previous experience.

Module Load Base is knowledge acquisition module which acquires knowledge from previously opened files. This module generates knowledge which is used for the definition of functional requirements.

Modules FR_Recog, DP_Recog, Analog $f$, and Construct are knowledge processors. Generally these modules consist of knowledge acquisition submodule for previously generated knowledge gathering, and recursive submodule for knowledge processing. Knowledge acquisition submodule organizes previously generated knowledge in the form of the list. Knowledge processing submodule takes the "head" of the list and processes it by the set of rules. The "tail" of the list is processed in the next recursive call. Knowledge generated in each recursive call is written on the knowledge transition file and available for use in the next recursive call as well as in the next knowledge processing modules. During the design process knowledge transition file is used for knowledge interchange.

First functional requirements are generated and written on the knowledge transition file. $D P$ recog is design parameters recognition module. Functional requirements recorded on knowledge transition file are first retrieved. Then recursive submodule of $D P$ recog which performs the mapping of functional requirements into design parameters based on analogy with previous projects is activated. 
Learning module includes deductive and inductive learning by analogy. Reasoning by analogy refers to previously designed projects. All existing similar FRs gained by experience in the allowed limits of error are retrieved and the list of machines $(\mathrm{Lm})$, as variant solutions for a given set of requirements, is formed. When the experience is not sufficient or the error is too large, the analogy is not applicable. Therefore, the module DP_recog has to go through the complete design procedure with all necessary computations.

Construction module uses knowledge generated by previous module. The construction module has two submodules: Couple_m and Schedule

The first submodule Couple $m$ integrates machine tools with the same or similar manufacturing tasks. The second submodule Schedule involves scheduling process based on time structure and information content related to this time structure (Babic, 1993).

Final design solution contains FMS layout structure and a schedule which is supposed to be optimal. The final solution is checked through simulation. Simulation module is universal data driven simulation system providing simulation of various FMS structures.

\section{APPLICATION OF FLEXY}

The established concept of intelligent system for FMS design was fully realized in the program package (FLEXY) developed in Prolog. For better understanding of proposed concept, the example of FMS design process using developed program package is given below.

The designing of the FMS for production of six machine tool components was the task given to the FLEXY. The parts and their operation sequences are given in Table 1.

Table 1 Operation sequences

\begin{tabular}{|c|c|}
\hline Part name & Operation sequence (operation name/operation time [min]) \\
\hline Gear box & hor. boring $/ 10$; hor. milling $/ 70 ;$ vert. milling $/ 10 ;$ cleaning $/ 1 ;$ inspection $/ 4$ \\
\hline Rotation table & vert. boring $/ 15$; vert. milling $/ 105 ;$ cleaning $/ 1$; inspection $/ 4$ \\
\hline Slider table & vert. milling $/ 50$; hor. boring $/ 10$; vert. milling $/ 60$; cleaning $/ 4$; inspection $/ 5$ \\
\hline Columns & vert. boring/20; vert. milling $/ 60$; hor. milling $/ 20 ;$ cleaning $/ 4$; inspection $/ 5$ \\
\hline Machine structure & $\begin{array}{l}\text { hor. milling } / 17 \text {; hor. boring } / 7 \text {; vert. milling } / 50 \text {; vert. milling } / 50 \text {; } \\
\text { cleaning } / 5 \text {; inspection } / 6\end{array}$ \\
\hline Bed ground & vert. milling/34; vert. milling/100; cleaning/2; inspection/5 \\
\hline
\end{tabular}

The first phase of the design process is FRs recognition. The FRs recognition module acquires knowledge about workpieces, process plans and requirements dealing with productivity and production costs. The FRs produced by this module expressed in Prolog have the following form:

FRs(M_Type,Acc,Gab,P_Op_L), where:

M_Type - Machining type required, Gab - Max machining dimension, Acc - Accuracy required, P_Op_L - List of parts and associated operation. FRs for the given example are given in Table 2 .

The second step is the generation of DPs. The DPs recognition module, uses the knowledge generated by previous module and the initial knowledge to produce a set of 
candidate machines for established FRs satisfaction. DPs expressed in Prolog have the following form:

where:

$$
\text { DPs(M,No,M_Acc,M_gab,P,Cph,M_P_Op_L) }
$$

$\begin{array}{llll}M & \text { - machine identification, } & \text { M_Acc } & \text { - machining accuracy, } \\ \text { No } & \text { - number of machines required, } & \text { M_gab } & \text { - max machining dimension, } \\ \text { P } & \text { - machine power, } & \text { Cph } & \text { - machine cost per hour, }\end{array}$

M_P_Op_L - list of parts where operations are performed on specified machine.

Table 2 Functional parameters obtained by FRs generation module

\begin{tabular}{|c|c|c|c|c|}
\hline$F R$ & M_Type & $A c c$ & Size & $P_{-} O p_{-} L$ \\
\hline FR1 & horizontal milling-boring & high & medium & [gear_box,1];[gear_box,2] \\
\hline FR2 & vertical milling-boring & normal & medium & [rotation table,1] \\
\hline FR3 & vertical milling-boring & $\begin{array}{l}\text { above } \\
\text { normal }\end{array}$ & medium & [gear_box,3];[rotation_table,2] \\
\hline FR4 & cleaning & - & medium & [gear_box,4];[rotation_table,3] \\
\hline FR5 & inspection & high & medium & [gear_box,5];[rotation_table,4] \\
\hline FR6 & horizontal milling-boring & normal & large & [columns,3] \\
\hline FR7 & vertical milling-boring & normal & large & $\begin{array}{l}\text { [slider_table, } 1 ; 2] ;[\text { columns, } 1] ;[\text { machine } \\
\text { _structure,3];[machine_structure }, 4]\end{array}$ \\
\hline FR8 & vertical milling-boring & high & large & [columns,2];[bed_ground,1] \\
\hline FR9 & horizontal milling-boring & high & large & $\begin{array}{l}\text { [slider_table,2];[machine_structure, } 1 ; 2] ; \\
\text { [bed_ground,2] }\end{array}$ \\
\hline FR10 & cleaning & - & large & $\begin{array}{l}\text { [slider_table, } 4] ;[\text { columns, } 4] \\
\text { [machine_structure,5];[bed_ground,3] }\end{array}$ \\
\hline FR11 & inspection & high & large & $\begin{array}{l}\text { [slider_table,5];[columns,5] } \\
\text { [machine_structure,6];[bed_ground,4] }\end{array}$ \\
\hline
\end{tabular}

The DPs obtained are given in Table 3. Information contents for candidate machines recognized by information content recognition module are also given in Table 3.

Finally, the construction module produces design solution with the aid of generated knowledge. By the use of minimal information content criterion (Axiom 2) the set of machines for FMS construction is made. Then the possible integrations of machining stations are done (machines with similar or equal performances are integrated if possible).

In general, FMS design is a coupled design because some DPs are satisfying more than one FRs (machines used for machining of more than one workpiece). It can be assumed, however, that if there is a production schedule the production of $n$-th part depends on the production of previous parts only. In that case FMS design is decoupled one. So, in the final step of FMS design process the optimal schedule providing decoupled design is generated. The design solution is represented in the form of the final set of DPs. DPs dealing with schedule (priority of workpiece machining) are added to the previous set of DPs. 
Design solution for the example is given in Table 4. The solution obtained was evaluated by simulation module of FLEXY system. The simulation results for designed FMS are given in Table 4. All FRs, including the one dealing with productivity, are met.

Table 3 Design parameters obtained by DPs recognition module

\begin{tabular}{llllllrl}
\hline FR & $M$ & No & M_acc & $P[k W]$ & $\begin{array}{l}\text { Cph } \\
{[\$ / h]}\end{array}$ & $\begin{array}{r}\text { Information } \\
\text { content }\end{array}$ & Machines selected \\
\hline FR1 & HMC60 & 0.6 & high & 4.0 & 3.0 & 8.59 & HMC60 \\
& HMC80 & 0.5 & high & 8.0 & 4.0 & 9.98 & \\
FR2 & VMC60 & 0.1 & high & 4.0 & 2.0 & 4.16 & VMC60 \\
& VMC80 & 0.1 & high & 8.0 & 3.0 & 941.7 & \\
& VMC70 & 0.1 & higher & 8.0 & 2.5 & 5.17 & \\
FR3 & VMC60 & 0.9 & high & 4.0 & 2.0 & 4.07 & VMC60 \\
& VMC80 & 0.8 & high & 8.0 & 3.0 & 1531.0 & \\
& VMC70 & 0.8 & higher & 8.0 & 2.5 & 8.64 & \\
FR4 & PCM & 0.1 & - & 2.0 & 1.5 & 6.12 & PCM \\
FR5 & CMM & 0.1 & high & 3.0 & 5.5 & 9.32 & CMM \\
FR6 & HMC60 & 0.2 & high & 4.0 & 3.0 & 3.91 & HMC60 \\
& HMC80 & 0.1 & high & 8.0 & 4.0 & 1409.0 & \\
FR7 & VMC60 & 1.8 & high & 4.0 & 2.0 & 18.22 & VMC60 \\
& VMC80 & 1.6 & high & 8.0 & 3.0 & 8086.0 & \\
FR8 & VMC60 & 0.7 & high & 4.0 & 2.0 & 8.52 & VMC60 \\
& VMC80 & 0.6 & high & 8.0 & 3.0 & 1328.8 & \\
FR9 & HMC60 & 1.0 & high & 4.0 & 3.0 & 20.03 & HMC60 \\
& HMC80 & 0.9 & high & 8.0 & 4.0 & 29.35 & \\
FR10 & PCM & 0.6 & - & 2.0 & 1.5 & 8.23 & PCM \\
FR11 & CMM & 0.2 & high & 3.0 & 5.5 & 13.95 & CMM \\
\hline
\end{tabular}

Table 4 Design solution

\begin{tabular}{crrl}
\hline Machine & No & Parts-operations list & $\begin{array}{l}\text { Machine } \\
\text { utilization }\end{array}$ \\
\hline HMC60 & 2 & $\begin{array}{l}\text { [slider_table,2];[machine_structure,1,2];[bed_ground,2]; } \\
\text { [gear_box,1,2];[columns,3] }\end{array}$ & $60 \%$ \\
VMC60 & 4 & $\begin{array}{l}\text { [columns,1,2];[bed_ground,1];[slider_table,1,3];[columns,1]; } \\
\text { [machine_structure,3,4];[rotation_table,1,2];[gear_box,3] }\end{array}$ & $93 \%$ \\
PCM & 1 & ALL WORKPIECES & $11 \%$ \\
CMM & 1 & ALL WORKPIECES & $20 \%$ \\
\hline
\end{tabular}




\section{CONCLUSION}

FLEXY is developed for the design of FMS as a complex manufacturing structure, but it can also be applied for the design of other, less complex, manufacturing systems.

Some existing expert systems for FMS design, as those described in (Fahmy, 1986) and (Lenz,1987), use simulation programs to generate experimental data for decision making. In these systems the designing process is iterative based mainly on data processing. One of essential advantages of the proposed system is the capability for learning, that is the gathering of experiences which results in a more efficient designing process based on knowledge processing.

FLEXY is verified in two different industrial environments. In both examples developed expert system offered significant advantages in comparison with the existing simulation packages. One of the examples is described in this paper.

The power of FLEXY is found in rich knowledge base achieved through many FMS design sessions. Computers with a rich knowledge base will have the ability to synthesize creative design. Further research is connected with the implementation of other varieties of inference (besides analogy-based inference).

The research presented in this paper is just a step in the development of a practical knowledge processing technology to meet challenges in the forthcoming knowledge-intensive industry.

\section{REFERENCES}

Babic B.R., (1993) Development of Intelligent System for Multipurpose FMS design, Ph.D. Theses, Belgrade University, Belgrade.

Fahmy, N.A. (1986) An Expert System for the Design of FMS Using Simulation Analysis, Ph.D. thesis, The University of Alabama,

Kim, S.J., Suh N.P (1991) Design of Software System Based on Axiomatic Design, Annals of the CIRP, Vol. 40/1/1991, 165-6.

Lenz, E. J. (1987) An Expert System for Manufacturing Simulation, in Modern Production Management Systems, (ed. Kusiak A.), Elsevier Science Publishers.

Milacic, V.R., Babic, B.R (1994) Concept of FMS Design Machine, Proceedings of The World Congress on Expert Systems, Lisbon, 658-8.

Russell, S.J. (1989) The Use of Knowledge in Analogy and Induction, Morgan Kaufman Publishers, Inc., San Mateo.

Suh, N. P (1990) The Principles of Design, Oxford University Press, New York.

Bojan R. Babic is senior researcher in Institute for Production Engineering and CIM at Faculty of Mechanical Engineering, Belgrade University. He received his Ph.D. degree from the University of Belgrade in 1993. Dr. Babic has been actively involved in the field of intelligent manufacturing systems research for over 10 years. His current research interests include artificial intelligence applications in manufacturing systems, machine learning and intelligent manufacturing systems. 